\title{
Ablation of caspase-1 protects against TBI-induced pyroptosis in vitro and in vivo
}

Wei Liu ${ }^{1,2^{*}+}$, Yuhua Chen ${ }^{1,2^{*+}}$, Jiao Meng ${ }^{1,2+}$, Minfei Wu ${ }^{3 \dagger}$, Fangfang Bi ${ }^{1,2}$, Cuicui Chang ${ }^{1,2}$, Hua Li ${ }^{2}$ and Liangjun Zhang ${ }^{2}$

\begin{abstract}
Background: Traumatic brain injury (TBI) is a critical public health and socioeconomic problem throughout the world. Inflammation-induced secondary injury is one of the vital pathogenic parameters of TBI. Molecular signaling cascades of pyroptosis, a specific type of cellular necrosis, are key drivers of TBI-induced inflammation.

Methods: In this study, mice with genetically ablated caspase-1 (caspase- $1^{-/-}$) were subjected to controlled cortical impact injury in vivo, and primary neuron deficient in caspase-1 through siRNA knockdown and pharmacologic inhibition was stimulated by mechanical scratch, equiaxial stretch, and LPS/ATP in vitro. We evaluated the effects of caspase-1 deficiency on neurological deficits, inflammatory factors, histopathology, cell apoptosis, and pyroptosis.

Results: During the acute post-injury period ( $0-48 \mathrm{~h}$ ) in vivo, motor deficits, anti-inflammatory cytokines (TGF- $\beta$ and IL-10), pro-inflammatory cytokines (IFN- $\gamma, \mathrm{IL}-1 \beta$, and IL-18), and blood lactate dehydrogenase (LDH), as well as pyroptosis-related proteins (caspase-1, caspase-1 fragments, caspase-11 and GSDMD), were increased. Caspase-1 was activated in the cortex of TBI mice. Inflammatory activation was more profound in injured wild-type mice than in caspase $-1^{-1-}$ mice. In vitro, mechanical scratch, equiaxial stretch, and LPS/ATP-induced neuron pyroptosis, apoptosis, $\mathrm{LDH}$ release, and increased expression of inflammatory factors. The effects of mechanical and inflammatory stress were reduced through inhibition of caspase-1 activity through siRNA knockdown and pharmacologic inhibition.
\end{abstract}

Conclusion: Collectively, these data demonstrate that pyroptosis is involved in neuroinflammation and neuronal injury after TBI, and ablation of caspase-1 inhibits TBI-induced pyroptosis. Our findings suggest that caspase-1 may be a potential target for TBI therapy.

Keywords: TBI, Inflammation, Neuroinflammation, Pyroptosis, Caspase-1, Neuron damage

\section{Background}

Traumatic brain injury (TBI) is a type of physical brain insult caused by blunt mechanical force [1]. According to the World Health Organization, TBI is a critical public health and socioeconomic problem throughout the world. Considering the incidence of TBI increases annually, TBI will be a major health problem and a substantial cause of disabilities by 2020 [2, 3]. Treatment of TBI can be very costly due to the need for on-going treatment of long-term effects, which can include psychiatric vulnerability and neurological disorders. The prevalence of neurological dysfunction following TBI has

\footnotetext{
* Correspondence: vincelau@sina.com; chenyuhua.358@163.com

${ }^{\dagger}$ Equal contributors

${ }^{1}$ Department of Medical Science Research Center, Peihua University, Xi'an

710125, People's Republic of China

Full list of author information is available at the end of the article
}

been increasingly appreciated $[4,5]$. For example, the risk of clinical depression is approximately1.5 times higher in TBI survivors compared to the general population. Furthermore, the risk for developing Alzheimer disease is 2.3 to 4.5 times greater, depending on whether the TBI is moderate or severe [6]. Worse, still no effective treatment options are available, and little is known about the complex cellular response to TBI.

TBI occurs when the brain is exposed to external forces that induce focal and/or diffuse injuries that include axonal shearing, edema, vascular damage, and neuronal death [7-9]. Injuries from TBI can range from mild to severe [10]. The post-TBI primary insult typically leads to secondary damage and neuronal death caused by inflammation $[8,9,11]$, oxygen radical formation $[12,13]$, calcium release [14], and mitochondrial dysfunction 
$[15,16]$. To date, TBI research outcomes have not translated into clinical therapeutic approaches [17]. Thus, there is an increasing need for further research into the signaling cascades activated by TBI.

Neuroinflammatory signaling leads to a specific type of inflammatory necrosis called pyroptosis, which is characterized by cell swelling, lysis, and release of proinflammatory cytokines and intracellular contents [18]. Pyroptosis is involved in pathological processes during viral or bacterial infection (or in the presence of products from pathogens such as lipopolysaccharides (LPS) or viral DNA) $[19,20]$, myocardial ischemia, lung and kidney injury, and cerebral stroke [21-24]. This type of inflammatory necrosis occurs predominantly in the phagocytes, macrophages, monocytes, and dendritic cells, as well as various other cell types such as $\mathrm{T}$ cells. The pro-inflammatory caspase subfamily, including caspase- 1 in humans and mice, caspase- 4 and caspase- 5 in humans, and caspase-11 in mice, mediate pyroptosis [19]. Li et al. found that chemical neurotoxicity-induced pyroptosis of the cortical neurons is dependent on caspase signaling through the Erk1/2-Nrf2/Bach1 signal pathway [25]. However, the specific mechanism of neuronal pyroptosis in TBI has not yet been elucidated.

To better understand the mechanism of brain injury responses, we used an in vivo mouse model of TBI and in vitro cellular models of mechanical stress and inflammation. Caspase-1 knockout (KO) mice (caspase- $1^{-1-}$ ), siRNA knockdown, and pharmacologic inhibition were used as tools to assess the involvement of caspase- 1 in downstream inflammation following TBI and mechanical stress. We found that genetic ablation, expression reduction, and pharmacologic inhibition of caspase-1 decreased TBI- and mechanical injury-induced behavioral neurological deficits, inflammatory cytokine protein and mRNA expression levels, and neuronal apoptosis. Ultimately, our results lead us to conclude that mechanical stress caused by TBI activates caspase- 1 which contributes to TBI-induced pyroptosis.

\section{Methods}

\section{Animals and TBI models}

In this study, we used caspase1-deficient ${\text { (caspase }{ }^{-/} \text {; }}^{-}$ Jackson Laboratories, Bar Harbor, ME, USA) adult male mice (8-10 weeks old) and age-matched C57Bl/6 (WT) male mice. Mice were housed in the animal care facility under a 12-h light-dark cycle, with ad libitum access to food and water. All surgical procedures were carried out in accordance with protocols approved by the Institutional Animal Care and Use Committee. The animals were anesthetized with intramuscular injection of chloral hydrate $(5 \%, 0.1 \mathrm{ml} / 10 \mathrm{~g})$. Mice were fixed in a stereotaxic frame, and the scalp was shaved and cleaned with iodophor. The left lateral aspect of the skull was exposed by retracting the skin and the surrounding soft tissue. Traumatic brain injury (TBI) was induced by dropping a 200-g steel weight with a flat end from a height of $5 \mathrm{~cm}$ onto the left lateral skull. The free-falling weight produced a moderate contusion injury of the left parietal cortex. Sham-injured control animals underwent a similar anesthesia protocol as the TBI animals with the exception that the scalp was not exposed and no weight was dropped. About $25-30 \%$ of the mice died in the first seconds after the trauma, but there was no delayed mortality or prostration in the surviving mice. The body temperatures of the animals were monitored by a rectal probe and fixed in a range of $37 \pm 0.5{ }^{\circ} \mathrm{C}$ using a heating pad. The animals were returned to their quarters after recovery from anesthesia and were allocated randomly into groups, with 18 mice in each group.

\section{Neurobehavioral training and evaluation}

Neurological deficits were assessed using well-established, modified neurological severity scoring (mNSS), open-field and Rotarod testing at 12, 24, and $48 \mathrm{~h}$ after TBI. Experimenters were blinded to the TBI or sham treatments as well as the genotype of the mice. Each behavioral test was repeated twice with four different trials to validate the data. The mNSS test consists of ten different tasks that can evaluate the motor (muscle status, abnormal movement), sensory (visual, tactile, and proprioceptive), balance, and reflex functions of mice. Neurological function was graded from 0 to $18(0=$ normal function; 18 =maximal deficit). One point was scored for each abnormal behavior or for the lack of a tested reflex. Therefore, higher scores imply greater neurological injury.

The open-field test was used to determine general activity levels and to measure anxiety-like behavior. Animals were monitored under moderate lighting for $15 \mathrm{~min}$ in a $40-\mathrm{cm}^{2}$ open field using video tracking software (ANY-Maze, Stoelting, USA). General activity was evaluated by determining the total of distance traveled. Anxiety-like behavior was assessed based on the pattern of exploration in the open field (center vs. periphery).

Fine motor coordination and learning were assessed using an accelerating Rotarod apparatus (RWD Life Science, China). On the day before the injury, mice were trained on the Rotarod for three consecutive trials at a slow rotational speed $(4 \mathrm{rpm} / \mathrm{min})$ for $1 \mathrm{~min}$ to adapt to the rod, followed by four additional trials with an accelerating rotational speed (from 4 to $40 \mathrm{rpm}$ in $5 \mathrm{~min}$ ) to obtain baseline latency. On each testing day, the mice were given four 300-s accelerating Rotarod trials with an inter-trial interval of $30 \mathrm{~min}$. The average latency to the first fall off the rod was recorded. Passive rotation, accompanying the rotating rod without walking, were also considered as a fall. 


\section{Primary neuronal culture and injury models}

The primary neuronal culture was prepared as follow: Cortex from embryonic day $14 \mathrm{BALB} / \mathrm{c}$ mice was used for cultures. After decapitation of the mice and removal of the meninges, the cortex was digested by papayotin, and the cells were treated with DNase I (Sigma) prior to centrifugation at $1000 \times g$ for $3 \mathrm{~min}$. Neurons were cultured on poly-L-lysine-coated dishes at a density of $1 \times 10^{6}$ cells per $10-\mathrm{cm}$ dish in minimum essential medium with $10 \%$ horse serum. The medium was changed to neuronal base medium (supplemented with 2\% B27, $0.2 \mathrm{mM}$ L-glutamine, and $1 \%$ penicillin-streptomycin) on the following day. The medium was changed every 3 days, and cultured cells were used for experiments on post-culture days 7-14. Subsequently, the primary neuron was collected and detected the Iba1 (microglia) and GFAP (astrocytes) expression through Western blotting (WB) and NeuN stain to assess neuronal purity.

We then performed sets of three experiments to examine the effects of mechanical and inflammatory stress on neurons in vitro: (1) neurons were incubation for $5 \mathrm{~h}$ with LPS and $5 \mathrm{mM}$ ATP for 30 mins; (2) neurons were scratched by a 200- $\mu$ l yellow gunshot; and (3) equiaxial stretch (12\% strain, $1.0 \mathrm{~Hz}$ frequency) was applied to cultured neurons for $4 \mathrm{~h}$, by a Flexcell ${ }^{\circ}$ FX $-5000^{\mathrm{Tm}}$ Tension System (Flexcell, USA). In the stretch model, neurons were inoculated in 6-well plates (BioFLEX ${ }^{\odot}$ ). At the same time, we studied the role of caspase- 1 in neuronal pyroptosis by caspase-1 siRNA knockdown or adding the caspase-1specific inhibitor, Belnacasan (VX-765, $10 \mu \mathrm{M}$, for $24 \mathrm{~h}$ ). After treatments, cells were used for flow cytometry, immunofluorescence, and protein or RNA extraction.

\section{Transient transfection}

The primary neuron was respectively infected with caspase-1 shRNA (m) lentiviral particles (Santa Cruz, sc29922-V) according to the manufacturer's protocol (Santa Cruz Biotechnology, Inc.). Plate targets cells in a 24-well plate $48 \mathrm{~h}$ prior to viral infection. The cells should be approximately $50 \%$ confluent before infection. Add $0.5 \mathrm{ml}$ of complete optimal medium with caspase- 1 shRNA lentiviral particles ( $\mathrm{MOI}=5$ ) (without polybrene), incubate cells for $48 \mathrm{~h}$, and then change complete optimal medium without lentiviral. At $72 \mathrm{~h}$ after infection, the RNAi efficiency was determined by qRT-PCR (Additional file 1: Table S1 and Additional file 2: Figure S1).

\section{Apoptosis analysis by Annexin V-PI double staining}

Neurons were stained with Annexin-V and propidium iodide (PI) using the Annexin-V-FITC kit (BD Biosciences) according to the protocol of the company. Briefly, cell culture medium was collected separately, and the treated cells were digested and washed twice with ice-cold PBS and re-suspended in the binding buffer at a concentration of $1 \times 10^{5}$ per $100 \mu \mathrm{l}$. Then, the cells were supplied with $5 \mu \mathrm{l}$ of Annexin V-FITC and $5 \mu \mathrm{l}$ of PI and incubated in the dark at RT for $10 \mathrm{~min}$. Next, $400 \mu \mathrm{l}$ of binding buffer was added into each sample for flow cytometry analysis (BD Accuri. C6 Plus, BD Biosciences).

\section{$\mathrm{LDH}$ release detection}

Mice were sacrificed under deep anesthesia for blood collection (centrifugation at $3000 \mathrm{~g}, 10 \mathrm{~min}$ ), and the blood serum was measured for lactate dehydrogenase $(\mathrm{LDH})$ release. For the primary neuron, supernatant from serum-free media was filtered using $0.2-\mu \mathrm{m}$ syringe filters to use for $\mathrm{LDH}$ release detection. The detection was using a commercially available kit (Solarbio, Beijing). One hundred microliters of the blood serum or supernatant was transferred to 96-well plates, and then the reaction mixture was added and incubated in the dark for $30 \mathrm{~min}$ at $\mathrm{RT}$. LDH concentration was quantified by measuring the absorbance at $490 \mathrm{~nm}$.

\section{Enzyme-linked immunosorbent assay}

Total protein concentrations were measured using a BCA Protein Assay Kit (Thermo Fisher Scientific, Carlsbad, CA, USA). The levels of TGF $\beta$, IFN- $\gamma$, IL-6, IL-10, IL-1 $\beta$, and IL-18 were measured using ELISA kits (Anoric-Bio, Tianjin, China) according to the manufacturer's instructions.

\section{Caspase- 1 activity detection}

The caspase- 1 activity was detected with Caspase- 1 Activity Assay Kit (Solarbio, Beijing). The specific steps were conducted according to the manufacturer's instructions: First, $2-10 \times 10^{6}$ cells were lysed in $50-100 \mu$ lysis buffer on ice for $10 \mathrm{~min}$. For tissue samples, 3-10 mg tissues were added to $100 \mu \mathrm{l}$ lysis and were homogenated with a tissue homogenizer and centrifuged. The supernatant was retained. Protein concentrations were detected using the Bradford method, ensuring that the protein concentration was $1-3 \mu \mathrm{g} / \mu \mathrm{l}$. A standard curve was prepared using the $p$ NA standard. Then, the optical density of specimen was read on a microplate reader (Molecular Device) at $405 \mathrm{~nm}$. The percentage of caspase-1 activity changes was calculated by the radio of OD405 of the experimental wells to that of the normal wells.

\section{TUNEL staining}

The cerebral cortex was harvested, and apoptosis was determined by terminal deoxynucleotidyl transferasemediated dUTP-biotin nick end labeling (TUNEL) staining, using a TUNEL Apoptosis Assay Kit (R\&D, Switzerland). TUNEL staining was performed with fluorescein-dUTP for apoptotic cell nuclei and 4',6diamidino-2-phenylindole (DAPI) to stain all cell nuclei. apoptosis index (AI), the number of TUNEL-positive cells 
divided by the total cells per field, was examined. Each AI was assessed in 20 randomly selected fields.

\section{Immunohistochemical analysis}

Mice were euthanized by injection of $5 \%$ chloralic hydras in accordance with our approved animal protocol, and the brain tissue was removed. The brain tissues were immediately perfused with $4 \%$ paraformaldehyde in PBS after removal and embedded in paraffin for sectioning. Following rehydration of the paraffin section and two washes in PBS, endogenous peroxidase activity was blocked using $3 \% \mathrm{H}_{2} \mathrm{O}_{2}$ for 10 min. Antigen crosslinking was conducted in an autoclave for $3 \mathrm{~min}$, and then the samples were washed twice in PBS. All preparations were then treated with goat serum blocking reagent (Abcam, ab7481) for $45 \mathrm{~min}$. Excess reagent was removed with a quick rinse with PBS. Sections were incubated with primary antibodies overnight. Following two washes in PBS, the secondary antibody (Abgent, ASS3403) were added to all slides for $30 \mathrm{~min}$. Excess reagent was removed, and the slides were washed in PBS and incubated with DAB (Solarbio, DA1010) for 5-10 min until the desired color appeared. All preparations were counterstained with hematoxylin (Solarbio, G1120) for 30 s. Protein expression was determined in five adjacent sections per sample.

\section{Western blot analysis}

Cells were lysed in lysis buffer containing Proteinase Inhibitor Cocktail (Roche Applied Sciences) and Halt Phosphatase Inhibitor Cocktail (Thermo Scientific). Protein concentrations were quantified by using a BCA protein kit (Thermo Scientific). Samples (50 $\mu \mathrm{g}$ protein per lane) were loaded on $12 \%$ SDS-PAGE gels. After electrophoresis, the proteins were transferred to nitrocellulose membranes. Then, the membranes were blocked with $5 \% \mathrm{BSA}$ and incubated at $4{ }^{\circ} \mathrm{C}$ overnight with the appropriate primary antibodies: caspase-1 (Abcam, 1:1000), caspase1 (p10) (AdipoGen, 1:1000), caspase-1 (p20) (AdipoGen, 1:1000), caspase-11 (Abcam, 1:1000), GSDMD (Santa Cruz, 1:100), Iba1 (Abcam, 1:1000), and GFAP (Abcam, 1:1000). Immunoreactivity was detected by incubation with horseradish peroxidase-conjugated secondary antibodies (Abgent, 1: 20,000) followed by chemiluminescent substrate development (Thermo Scientific, USA). Optical densities of the bands were calculated using a MiVnt image analysis system (Bio-Rad, CA, USA). All samples were run in parallel with four replicates.

For supernatant detection, supernatant from neurons in serum-free media was filtered using $0.2-\mu \mathrm{m}$ syringe filters and concentrated using Centricon Plus-20 centrifugal filter devices (Millipore). Concentrated supernatant (50 $\mu \mathrm{g}$ protein per lane) was separated by $15 \%$ SDS-PAGE and transferred to nitrocellulose membranes, and processing was assessed by Western blotting using anti-caspase-1 (Abcam, 1:1000), anti-caspase1 (p10) (AdipoGen, 1:1000), and caspase-1 (p20) (AdipoGen, 1:1000). Uncropped blots for the pro-form and cleaved fragments of caspase-1 in figures were supplemented in Additional file 3: Figure S2.

To extract protein from the cortical brain tissues, the frozen brain samples were homogenized in ice-cold buffer containing $20 \mathrm{mM}$ HEPES ( $\mathrm{pH} 7.0$ ), $150 \mathrm{mM}$ $\mathrm{NaCl}, 1 \mathrm{mM}$ EDTA, 1\% Triton X-100, Proteinase Inhibitor Cocktail (Roche Applied Sciences), and Halt Phosphatase Inhibitor Cocktail (Thermo Scientific) for $30 \mathrm{~s}$. The samples were sonicated through the Cell Ultrasound Cruizer (Thermo Scientific) for $10 \mathrm{~s}$ and centrifuged at $15,000 \mathrm{rpm}$ for $10 \mathrm{~min}$, and total proteins were recovered in the supernatants.

\section{Immunofluorescence assays}

Primary neurons were subjected to immunofluorescence by the protocol described below. Cells were fixed by $4 \%$ PFA for $20 \mathrm{~min}$ at RT followed by PBS wash for three times ( 5 min each). The cells were then incubated by the blocking buffer $(0.2 \%$ TritonX-100 and 5\% BSA dissolved in PBS) for $1 \mathrm{~h}$. After PBS wash for three times, the cells were then incubated with the primary antibodies of NeuN (Abcam, 1:300,), dissolved in the blocking buffer at $4{ }^{\circ} \mathrm{C}$ for $24 \mathrm{~h}$. PBS wash was carried out followed by the treatments with the Alexa Fluor 488/568-conjugated secondary antibody (Abgent, 1:1000) dissolved in the blocking buffer for $2 \mathrm{~h}$ at RT. After incubation with secondary antibodies, cells were washed and counterstained with DAPI solution $(1 \mu \mathrm{g} / \mathrm{ml}$ in the blocking buffer $)$ for $10 \mathrm{~min}$ at RT. Cells were analyzed by fluorescence microscope (Leica, IX71).

\section{Quantitative real-time PCR}

Total RNA was isolated from the cortical brain tissue or primary cultured neurons, lysed in Trizol reagent (Invitrogen) to recover the total RNA according to the manufacturer's protocol (Trizol ${ }^{\mathrm{Tm}}$ Reagent, Invitrogen). The total RNA was reversely transcripted to cDNA using a HiFi-MMLV cDNA First Strand Synthesis Kit (CW Bio, China). Quantitative real-time PCR was performed using GoTaq qPCR Master Mix (Promega) on the CFX96TM Real-Time System (Bio-Rad). GAPDH was amplified in parallel as an internal control. Primer sequences as follow, TLR4 F: 5' - TCACAACTCGCCCAAGGAGGAA -3', R: 5' - AAGAGACCACGGCAGAAGCTAG -3'; MyD88 F: 5' - CCACCTGTAAAGGCTTCTCG -3', R: 5' - CTAGAG CTGCTGGCCTTGTT -3'; NLRP3 F: 5' - GCTAAGAAGGACCAGCCAGAGT- 3', R: 5' - GAACCTGCTTCTCAC ATGTCGT -3'; GAPDH: F: 5' - AACTTTGGCATTGTGGAAGG -3' R: 5' - GGATGCAGGGATGATGTTCT -3'. 


\section{Statistical analysis}

All the data were presented as the mean \pm SEM and were analyzed using SPSS statistical software (version 22.0, IBM). Non-parametric data from the mNSS test were analyzed using the Kruskal-Wallis $H$ analysis followed by a Mann-Whitney $U$ test. One-way analysis of variance (ANOVA) with repeated measures was used to analyze Rotarod data. The remaining biochemical data were analyzed using a two-way ANOVA. Each experiment was repeated three times, and significance was determined with two-tailed Student's $t$ test or one-way ANOVA. Error bars represent \pm SEM. $p$ value lower than 0.05 was considered as significant.

\section{Results}

\section{TBI-induced neurological deficits}

To assess neurological deficits post-TBI, we conducted a time course of behavioral testing before TBI and 12, 24, and $48 \mathrm{~h}$ following TBI. Prior to surgery, there was no difference in mNSS and Rotarod scores between the groups of mice. In the mNSS test (Fig. 1a), mice in the sham group showed no significant differences at 12, 24, and $48 \mathrm{~h}$; however, TBI caused significantly higher mNSS relative to the sham group $(p<0.001)$. The mNSS scores were maximal at $24 \mathrm{~h}$ and gradually decreased, albeit insignificantly, with time in TBI group. The scores remained significantly different between the TBI and sham groups at $48 \mathrm{~h}$ post-injury $(p<0.05)$. Caspase- $1^{-/-}$-TBI mice exhibited lower mNSS compared to the wide-type (WT) mice subjected to TBI (WT-TBI) $(p<0.01)$. To examine spontaneous locomotor activity in response to a novel environment, all mice were tested in the open-field behavioral task (Fig. 1c). WT-TBI mice spent a shorter time in the perimeter zone and traveled less total distance than sham group $(p<0.01)$. A modest yet statistically significant difference in perimeter zone and total travel distance was revealed between WT-TBI and caspase- $1^{-/-}$-TBI mice $(p<0.05)$. In the Rotarod test (Fig. 1b), the sham groups exhibited the best performance relative to the TBI groups $(p<0.001)$. The scores were minimal at $24 \mathrm{~h}$ and following gradually increased with time in WT-TBI group. Caspase-1 $1^{-/}-$TBI mice had higher scores relative to the WT-TBI group $(p<0.05)$. The brains of the mice were assessed postmortem. TUNEL staining
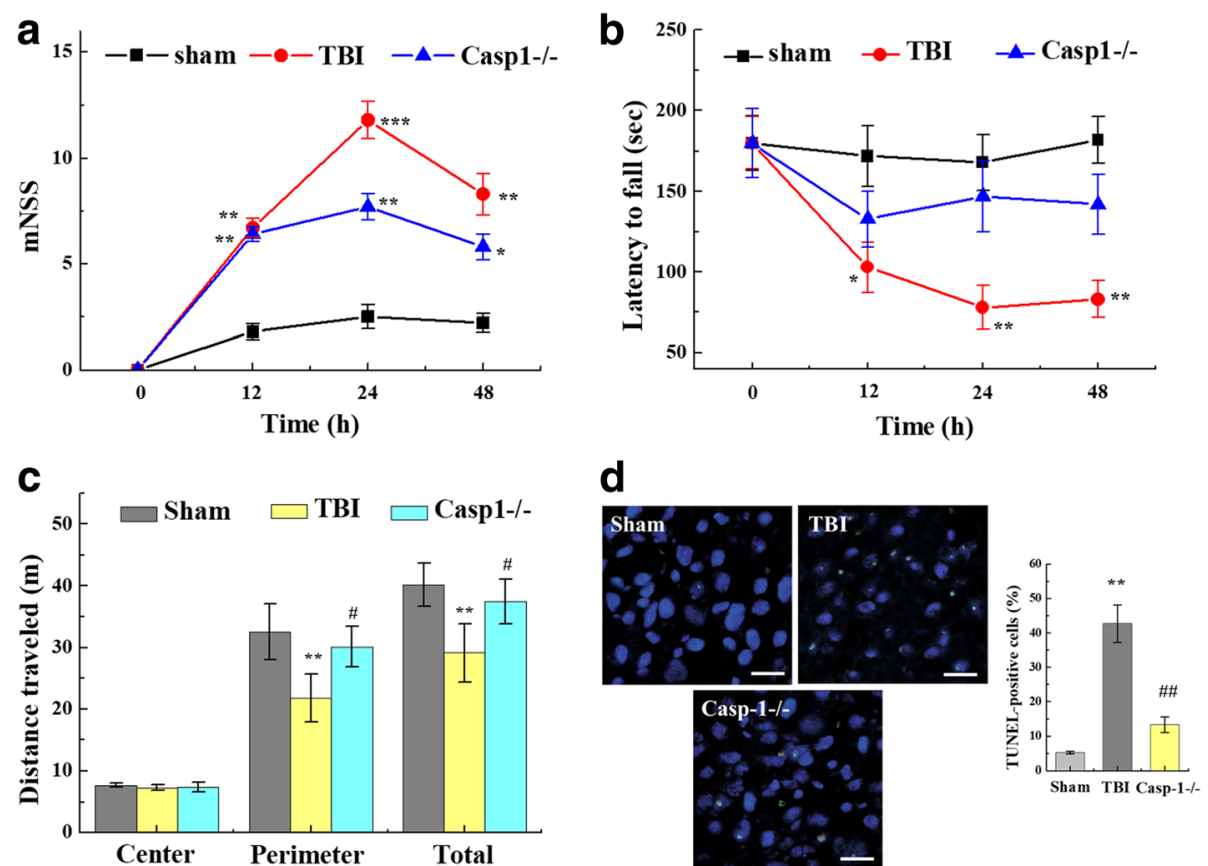

Fig. 1 Neurological deficits after TBI. Neurological effects of TBI were assessed by a mNSS, b Rotarod, and c open-field behavioral task tests prior to and 12,24 , and $48 \mathrm{~h}$ post-TBI. a mNSS scores were significantly higher in the TBI groups relative to those in the sham groups. The mNSS scores were maximal at $24 \mathrm{~h}$ and gradually decreased with time in the TBI group. No significant difference was observed between the caspase- ${ }^{-1-}$ and WT-TBI group at $12 \mathrm{~h}$. However, the caspase-1 ${ }^{-1-}$ group showed significantly lower mNSS scores relative to the WT-TBI group at 24 and $48 \mathrm{~h}$ post-TBI. b Rotarod scores were minimal at $24 \mathrm{~h}$ and gradually increased with time in WT-TBI group. Relative to WT-TBI mice, TBI-induced impaired Rotarod performance was attenuated in the caspase-1 ${ }^{-1-}$ mice at 24 and $48 \mathrm{~h}$ after TBI. $\mathbf{c}$ In the open-field task, all mice traveled the same distance in the center zone compared to the perimeter zone. WT-TBI mice spent less time in the perimeter zone of the open-field chamber compared to caspase- $1^{-/-}$-TBI mice. Total travel distance was significantly different between WT-TBI and caspase- $1^{-/-}$-TBI mice. $\mathbf{d}$ TUNEL staining was used to detect cortical damage in our TBI model. Apoptosis index, the number of TUNEL-positive cells divided by the total cells per field, was examined. Each Al was assessed in 20 randomly selected fields. Data are presented as the mean \pm SEM. ${ }^{* *} p<0.01$ and ${ }^{* * *} p<0.001$ versus sham group, $\# p<0.05$ and \#\#p<0.01, compare to TBI group $(n=11)$ (mNSS, Rotarod, and open-field) 
was used to detect cortical damage. The green fluorescence was dramatically enhanced in WT-TBI group compared to the sham group $(p<0.001)$. Apoptosis was significantly reduced in the brains of caspase- $1^{-1-}$-TBI mice relative to the WT-TBI group ( $p<0.05$; Fig. 1d).

\section{Inflammatory cytokine levels in the cortex following TBI}

To compare the protein levels of inflammatory-associated mediators in the cortex, an array of inflammatory cytokines were measured using ELISA. All detected cytokine levels were the lowest in the sham groups compared to the other groups (Fig. 2). Protein levels of the anti-inflammatory cytokines TGF- $\beta$ at $12 \mathrm{~h}(p<0.01)$, $24 \mathrm{~h}(p<0.001)$, and $48 \mathrm{~h}(p<0.001)$ and levels of IL-10 at $12 \mathrm{~h}(p<0.001), 24 \mathrm{~h}(p<0.001)$, and $48 \mathrm{~h}(p<0.001)$ post-TBI increased in the WT-TBI group compared to the sham group (Fig. 2a, b). Furthermore, levels of the pro-inflammatory cytokines IFN- $\gamma$, IL-1 $\beta$, and IL-18 were all augmented significantly at $24 \mathrm{~h}(p<0.001)$ and $48 \mathrm{~h}(p<0.01)$ in the TBI group relative to the sham group
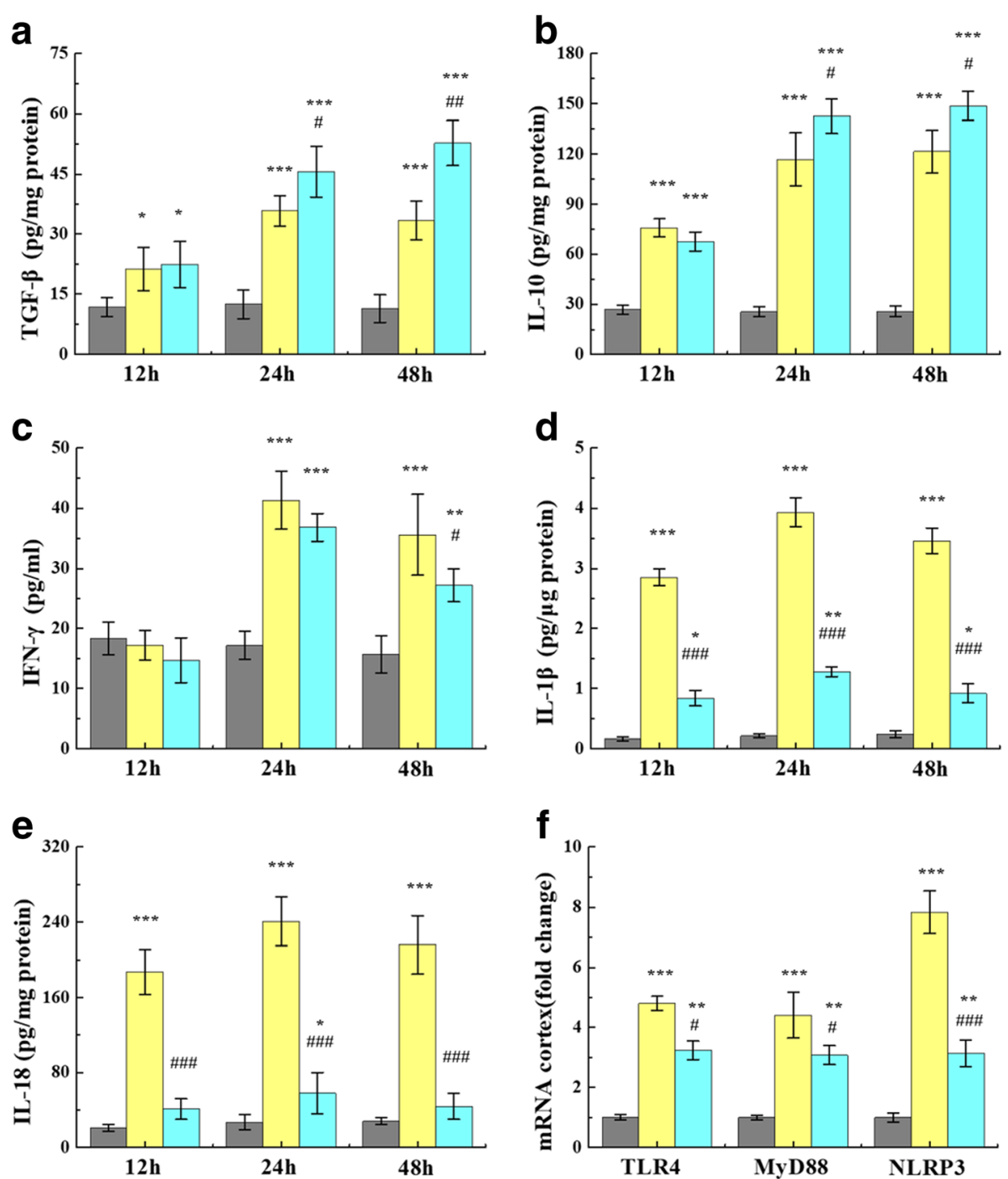

Sham

TBI

Casp1-/-

Fig. 2 Inflammatory cytokine expression in the cortex following TBI. a-e Relative to the sham group, concentrations of the anti-inflammatory cytokines $\mathbf{a}$ TGF- $\beta 1$ and $\mathbf{b} \mathrm{IL}-10$ and pro-inflammatory cytokines $\mathbf{c}$ IFN- $\gamma, \mathbf{d} I \mathrm{~L}-1 \beta$, and $\mathbf{e} \mathrm{IL}-18$ were significantly increased in the region of the contusion at 12, 24, and $48 \mathrm{~h}$ after TBI, whereas IFN- $\gamma$ level barely had changed at $12 \mathrm{~h}$ after TBI. Caspase- ${ }^{-1-}$-TBI mice had a less dramatic increase in anti-inflammatory cytokines and pro-inflammatory cytokines than the WT-TBI mice. $\mathbf{f}$ mRNA expression of inflammatory cytokines, TLR4, MyD88, and NLRP3, at $24 \mathrm{~h}$ post-TBI. TBI induced a marked increase in TLR4, MyD88, and NLRP3 mRNA expression in the injured WT-TBI mice brains relative to the sham group, but caspase- $1^{-1-}$ only exhibited mild increases. Data are presented as the mean \pm SEM. ${ }^{* *} p<0.01$ and ${ }^{* * *} p<0.001$, compared to sham group, \#p $<0.05$ and \#\#\# $<0.001$ versus TBI, $n=6 /$ group 
(Fig. 2c-e). Meanwhile, the levels of these proteins, IFN- $\gamma$, IL-1 $\beta$, and IL-18, peaked at $24 \mathrm{~h}$ and declined significantly by $48 \mathrm{~h}(p<0.05$, relative to $24 \mathrm{~h})$. Post-TBI at $24 \mathrm{~h}$, protein levels of TGF $\beta$ and IL-10 significantly increased in injured caspase- $1^{-/-}$mice as compared to WT-TBI $(p<0.05)$. IFN- $\gamma$ in caspase- $1^{-1-}-$ TBI mice reduced as compared to WT-TBI group $(p<0.05)$ at $48 \mathrm{~h}$. There was no significant difference in protein levels of IL-1 $\beta$ and IL-18 in caspase- $1^{-/-}$-TBI mice compared to sham. Gene expression related to the inflammation in the cortex region of the brain tissues was analyzed using qRT-PCR. Consistent with the ELISA results, TBI induced dramatic increases in mRNA expression of inflammatory cytokines compared to the sham groups at $24 \mathrm{~h}$ post-injury. The mRNA expression levels of TLR4, MyD88, and NLRP3 all ascended at $24 \mathrm{~h}$ post-TBI $(p<0.001)$ (Fig. 2f). However, NLRP3 mRNA was dramatically reduced in caspase-1 -l--TBI mice as compared to WT-TBI group $(p<0.001)$.

\section{TBI-induced pyroptosis in mice model}

To determine whether pyroptosis was occurring in the murine TBI model and presented time-dependence, inflammation was assessed in the cortex by WB, caspase-1 activity assay kit, and immunohistochemistry. As shown in Fig. 3a, WB assay was performed to quantify pyroptosis-related protein expression at $0,1,2,4,8,12$, and $24 \mathrm{~h}$ post-TBI. Caspase- $1 \mathrm{p} 45$ was elevated at $8 \mathrm{~h}$ (1.4-fold), $12 \mathrm{~h}$ (1.9-fold), and $24 \mathrm{~h}$ (2.0-fold) compared to the brain tissue from uninjured animals. The p20 and p10 fragments of caspase- 1 increased gradually- $24 \mathrm{~h}$ to 5.6-folds and 1.5-folds, respectively. Meanwhile, caspase-11 and GSDMD protein expression increased after TBI. At $24 \mathrm{~h}$ post-TBI, caspase-11 exhibited a 3.5-fold increase, and GSDMD expression increased 3.6-fold relative to normal brain tissue. As shown in Fig. 3b, caspase-1 (including p10, p20, and p45), caspase-11, and GSDMD expression were a significant reduction in caspase$1^{-1-}$-TBI mice when relative to WT-TBI mice at $24 \mathrm{~h}$. Immunohistochemistry was used to detect caspase-1, caspase-11, and GSDMD protein distribution in the trauma region and the surrounding brain tissue at $24 \mathrm{~h}$ post-TBI. As shown in Fig. 3c, caspase- 1 and caspase- 11 were mainly expressed in the injured area in WT-TBI mice, while they were less expressed in caspase- $1^{-/-}$-TBI mice. They had a high degree of co-localization and gradually diffused outward radially to the surrounding tissue, and the expression gradually decreased in a radial distribution from the injury to the surrounding area. Consistent with the WB results, caspase- 1 activity significantly changed after TBI. Following TBI, caspase- 1 activity was significantly greater in cortical samples from TBI animals than non-injured animals (Fig. 3d). For instance, at $24 \mathrm{~h}$ post-injury, caspase- 1 had 15.4-fold greater activity in WT-TBI mice as compared to sham controls. The caspase- 1 activity was less affected in the caspase- $1^{-/}$-TBI mice. At $24 \mathrm{~h}$ post-injury, the caspasedeficient mice exhibited only a 2.3-fold increase in the activity relative to the normal brain tissue (Fig. $3 \mathrm{~d}$ ). $\mathrm{LDH}$ is found in almost all body tissues. It plays an important role in cellular respiration, the process by which glucose from food is converted into usable energy for cells. Although $\mathrm{LDH}$ is abundant in tissue cells, blood levels are normally low. While the tissues are damaged by injury, they release more LDH into the bloodstream. Interestingly, serum LDH was tested at $24 \mathrm{~h}$ following TBI, since cerebral injury increases cellular LDH leakage. Serum LDH concentration increased approximately 2.0-fold in WT-TBI mice relative to the sham group $(p<0.001)$ and was near normal levels in caspase-1 $1^{-1-}$-TBI mice relative to the WT-TBI mice $(p<0.01)$ (Fig. 3e).

\section{Neuron injury-induced apoptosis}

Primary neuron culture was potential contamination with another type of cells, such as glial cells. The primary neuron was collected and detected the Iba1 (microglia) and GFAP (astrocytes) expression through WB to assess neuronal purity. At 7 days, Iba1 and GFAP were expressed, and they were disappeared following culture at 10 and 14 days (Fig. 4a). At 10 days, there were no obvious protein bands of Iba1 and GFAP, when 30, 50, and $70 \mu \mathrm{g}$ protein were loaded respectively (Fig. 4b). Furthermore, we have also stained the primary culture cells by markers for neurons (NeuN) and confirmed that more than $90 \%$ of cells were neurons (Fig. 4c).

In order to continue to explore the correlation between neuron damage and pyroptosis, we stimulated cultured primary neuron mechanically with scratch and stretch or chemically with LPS/ATP treatment. Apoptosis was detected by an Annexin-V-FITC kit. To validate these methods of mechanical and chemical injury, we assessed the apoptosis rate over time. Scratch, stretch, and LPS/ ATP all significantly induced apoptosis of primary neuron (Fig. 5a). Apoptosis scores were $47.14 \pm 5.51$ for scratch stimulation, $46.56 \pm 3.54$ for equiaxial stretch, and $45.37 \pm 3.87$ for LPS/ATP stimulation. The culture supernatant was collected to detect $\mathrm{LDH}$ release by scratch, stretch, and LPS/ATP stimulation. Supernatant LDH concentration increased approximately 1.7-fold, 1.6-fold, and 2.2-fold by scratch, stretch, and LPS/ ATP stimulation, respectively, relative to normal group $(p<0.001)$ (Fig. 5b).

\section{Neuron injury potentially induced inflammation and pyroptosis}

ELISA and qRT-PCR were used to determine the expression of inflammatory mediators following scratch, stretch, or LPS/ATP stimulation of primary neuron. Scratch, stretch, and LPS/ATP stimulation significantly increased secretion of IFN- $\gamma$, IL- 6 , IL- $1 \beta$, and IL-18 compared to 
a

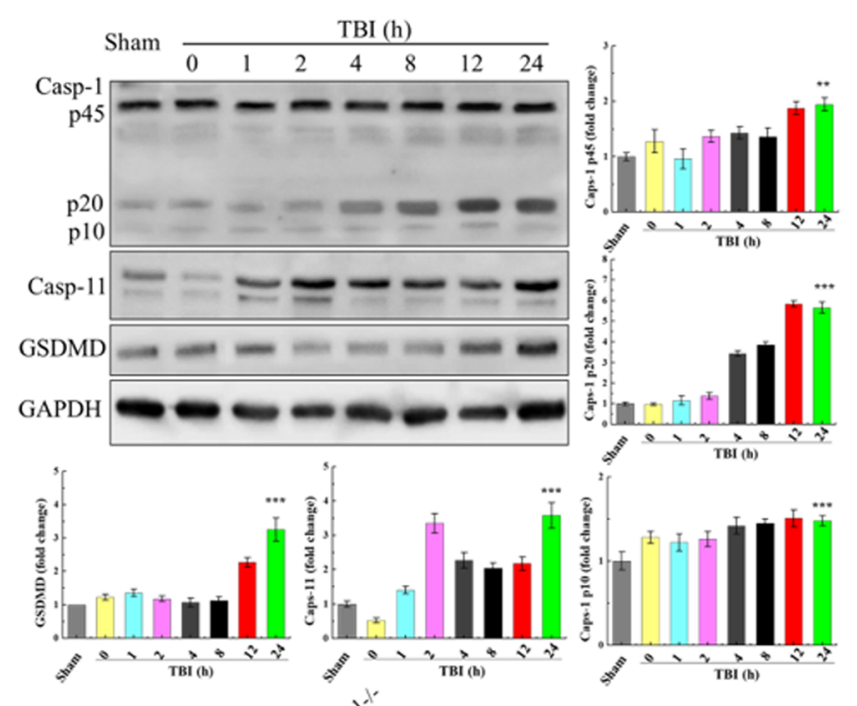

b
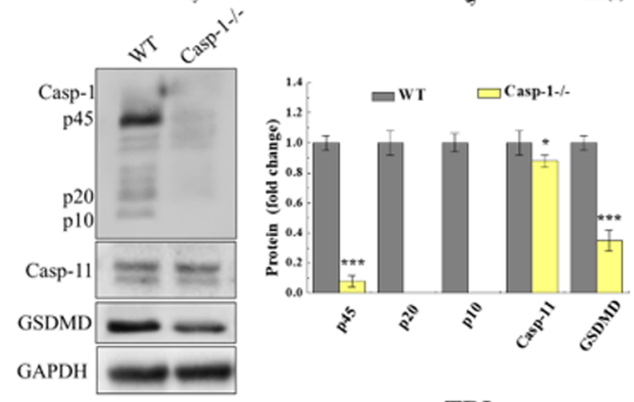

C

Sham

TBI
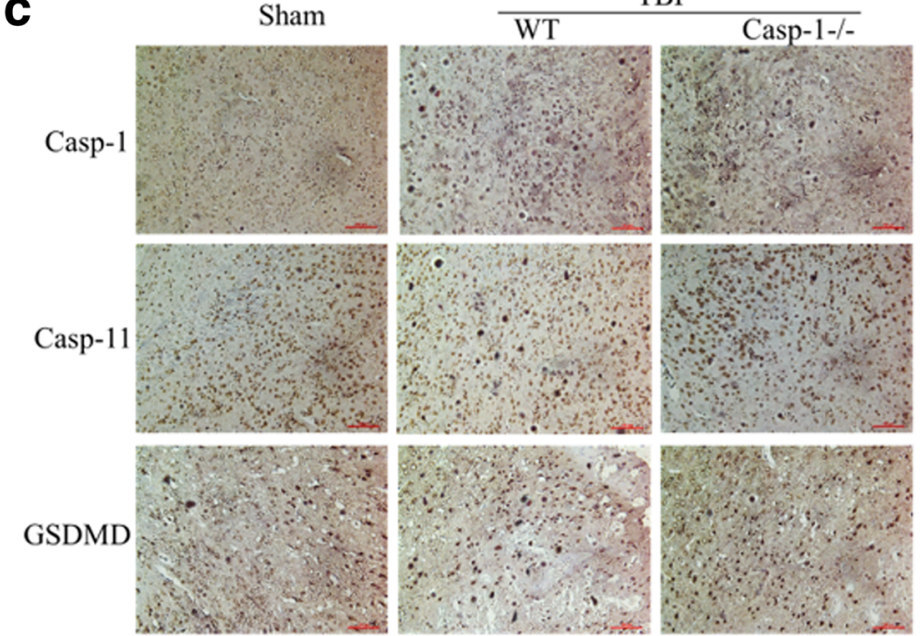

d

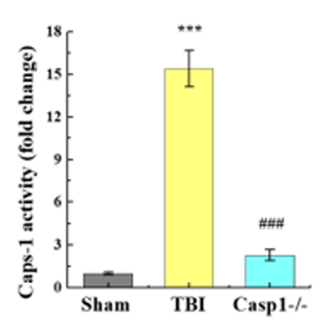

e

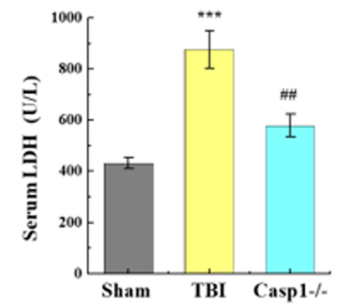

Fig. 3 (See legend on next page.) 
(See figure on previous page.)

Fig. 3 Pyroptosis in the murine model of TBI. a Expression of pyroptosis-related proteins in response to TBI was assessed by Western immunoblotting (WB). After 0, 1, 2, 4, 8, 12, and 24 h post-TBI, caspase-1, caspase-11, GSDMD, and caspase-1 protein fragments were severally elevated in WT-TBI mice compared to sham controls. All data are expressed as means \pm SD, $n=5$. Post-TBl at $24 \mathrm{~h}$, caspase $\mathbf{1}^{-1-}$ mice exhibited reduced TBl-induced pyroptosis. b In caspase-1 ${ }^{-1-}$ mice, pyroptosis-related proteins were less prominent than the WT-TBI group, which demonstrated in WB results. All data are expressed as means \pm SEM,$n=5$. c Micrograph at $\times 20$ magnification of caspase-1, caspase-11, and GSDMD immunostaining in the cortex in the vicinity of the lesion. As shown in the immunohistochemistry results, caspase-1, caspase-11, and GSDMD distributed in trauma organization and surrounding. The cortex showed a strong increase in caspase-1 and GSDMD staining in the WT-TBI compared to caspase- $1^{-/-}-\mathrm{TBI}_{\mathrm{I}}$ at $24 \mathrm{~h}$ post-TBI. However, caspase-11 was no obvious difference between caspase-1-1--TBI and WT-TBI. One representative experiment of four was shown. d Caspase-1 activity was stimulated after TBI. In contrast, TBI-induced increase in caspase-1 activity was significantly reduced in caspase- $1^{-/-}$mice (\#\#\#p < 0.001, WT-TBI vs. caspase-1 $1^{-1-}$-TBI). e Serum LDH was tested at $24 \mathrm{~h}$ following TBI since cerebral injury increases cellular LDH leakage $(p<0.001)$. Data are presented as the mean \pm SEM. ${ }^{*} p<0.05$ and ${ }^{* * *} p<0.001$ versus sham group, \#\#\# $<0.001$ versus TBI group, $n=6$

untreated neurons $(p<0.001$; Fig. $6 \mathrm{a}-\mathrm{d})$. IL-1 $\beta$ was secreted at $12.76 \pm 1.37 \mathrm{pg} / \mathrm{ml}$ from untreated neurons and at levels of $491.54 \pm 56.33 \mathrm{pg} / \mathrm{ml}$ (scratch), 424.86 $\pm 71.36 \mathrm{pg} / \mathrm{ml}$ (equiaxial stretch), and $825.63 \pm 51.16 \mathrm{pg} /$ $\mathrm{ml}$ (LPS/ATP) in the stimulation groups. IL-18 was secreted at $12.14 \pm 1.81 \mathrm{pg} / \mathrm{ml}$ from untreated neurons, whereas stimulation caused increases in levels to $74.52 \pm 5.33 \mathrm{pg} / \mathrm{ml}$ (scratch), $83.42 \pm 5.36 \mathrm{pg} / \mathrm{ml}$ (equiaxial stretch), and $123.67 \pm 7.16 \mathrm{pg} / \mathrm{ml}$ (LPS/ATP). TLR4, $M y D 88$, and NLRP3 mRNA were analyzed using qRTPCR (Fig. 6e). Neuron injury from scratch, stretch, and LPS/ATP stimulation induced dramatic increases in the expression: TLR4 mRNA increased 4.5-fold, $M y D 88$ mRNA 4.0-fold, and NLRP3 mRNA 9.0-fold relative to untreated neurons (Fig. 6e). Expression of NLRP3 was remarkably higher than TLR4 and MyD88 $(p<0.001)$. Significant changes in caspase- 1 activity were detected using the Caspase-1 Activity Assay Kit, and pyroptosisrelated protein expression was also detected using a WB assay. Primary neuron was lysed to detect caspase-1 activity after scratch, stretch, or LPS/ATP stimulation. Caspase-1 activity was increased, respectively, 10.3-, 12.4-, and 21.6-fold relative to normal culture $(p<0.001)$ (Fig. 6f). In separate samples, WB assays were performed to quantify pyroptosis-related protein expression. As shown in Fig. 6g, mechanical scratch, equiaxial stretch, and LPS/ATP stimulation enhanced the expression of caspase-1, caspase-11, and GSDMD. Moreover, there leaked a large number of caspase-1 p45 and its fragments (p10 and p20) in the culture supernatant.

\section{Caspase-1 effected neuron injury}

In order to determine whether caspase- 1 resides in upstream cellular signaling pathways that lead to pyroptosis after mechanical and chemical stress and injury, we utilized caspase-1 siRNA to knockdown expression of caspase-1 as well as a pharmacological inhibitor, VX-765. Caspase-1 siRNA and inhibition with VX-765 reduced neuron apoptosis following mechanical scratch, equiaxial stretch, and a

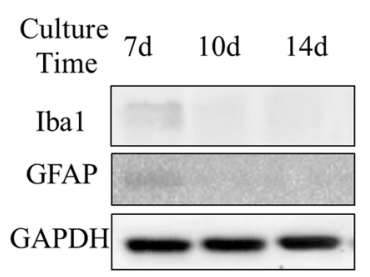

b

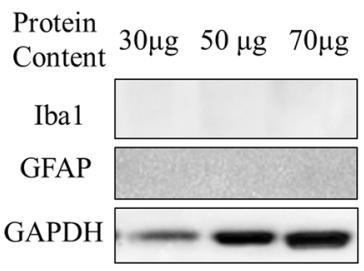

C
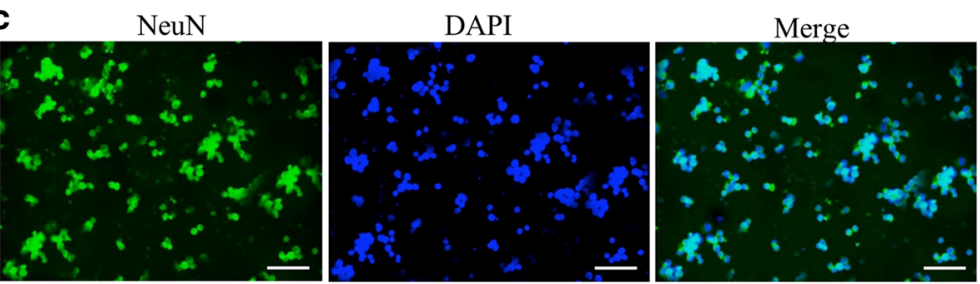

Fig. 4 The purity of in vitro primary neuron culture. a Primary neuron was collected at 7, 10, and 14 days, respectively; $50 \mu g$ protein per lane was used to detect Ibal and GFAP expression. At 7 days, Ibal and GFAP were expressed, they were disappeared following culture at 10 and 14 days. b 30, 50, and $70 \mu \mathrm{g}$ protein per lane were used to detect lba1 and GFAP expression at 10 days. As before, there was no obvious protein bands of Iba1 and GFAP. c Primary neurons were stained by the neuronal marker NeuN at 10 days. To confirm the purity of in vitro primary neuron culture, cells were stained by NeuN, and DAPI was used to counterstain the cellular nucleus. Almost all cells were stained by NeuN. One representative experiment of four was shown 

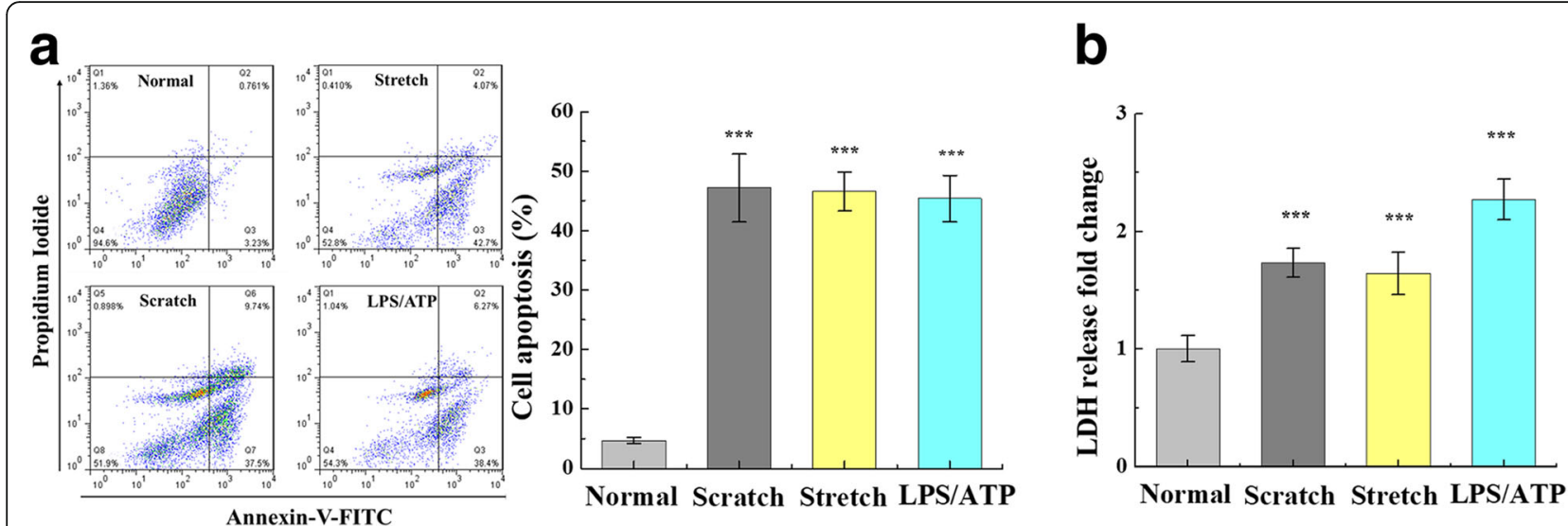

Fig. 5 Neuron injury-induced apoptosis in cultured primary neuron. a Flow cytometer results for apoptosis following scratch-, stretch-, and LPS/ ATP-induced neuron damage. Scratch, stretch, and LPS/ATP stimulation induced significant apoptosis in cultured primary neuron $\left({ }^{* * *} p<0.001\right.$, relative to the normal group). b Culture supernatant was collected to detect LDH release by scratch, stretch, and LPS/ATP stimulation. Supernatant LDH concentration increased by scratch, stretch, and LPS/ATP stimulation $(p<0.001)$. All data are expressed as means \pm SEM; one representative experiment of five was shown

LPS/ATP stimulation (Fig. 7a). After VX-765 treatment, apoptosis rates were $26.36 \pm 3.16$ cells/h for scratch, $23.13 \pm 2.13$ cells $/ \mathrm{h}$ for equiaxial stretch, and $21.79 \pm 1.51$ cells/h for LPS/ATP. Similarly, with siRNA knockdown of caspase-1, apoptosis scores were $23.54 \pm 3.11$ cells/h for scratch, $16.82 \pm 1.28$ cells/h with equiaxial stretch, and $17.72 \pm 1.11$ cells/h with LPS/ATP. In comparison, the apoptosis scores for neurons with fully functional caspase-1 were significantly greater-41.36 \pm 6.44 cells $/ \mathrm{h}$ for scratch, $47.38 \pm 4.61$ cells $/ \mathrm{h}$ for equiaxial stretch, and $44.62 \pm 4.81$ cells/h for LPS/ATP. The culture supernatant was collected to detect LDH release. Supernatant $\mathrm{LDH}$ concentration observably increased after the scratch, stretch, or LPS/ATP stimulation, relative to normal culture $(p<0.01)$, whereas the leaked LDH significantly reduced in caspase-1 siRNA and VX-765 groups, relative to control group $(p<0.05)$ (Fig. 7b). Enhanced caspase-1 activity by neuronal injury was inhibited by caspase-1 siRNA and VX-765 inhibition (Fig. 7c). Expression of caspase-1, caspase-11, and GSDMD decreased in caspase-1 siRNA and VX-765 groups, and caspase-1 p45 and its fragments (p10 and p20) significantly reduced in the culture supernatant, simultaneously (Fig. 7d). In order to demonstrate the reduction in the pro-form of caspase-1 levels by VX-765 was not an artifact due to direct VX-765 neurotoxicity, VX-765 groups, and a vehicle control (DMSO as the menstruum) for VX765 treated with neuron. CCK 8 results demonstrated that there was no neurotoxicity by VX-765 at $10 \mu \mathrm{M}$ (Additional file 4: Figure S3). Further, ELISA results showed decreased secretion of neuroinflammatory mediators, such as IL-1 $\beta$ and IL-18, when the caspase-1 was inhibited (Table 1).

\section{Discussion}

Results of this study demonstrate that pyroptosis modulates responses to the acute phase of TBI through caspase-1. Caspase-1 is expressed and activated in neurons and therefore is likely to be activated via the mechanical damage. During the acute post-traumatic period (0-48 h), neurological deficits, inflammatory cytokine, and pyroptosis are greater in the brain-injured mice. To date, there are scant data regarding the regulating of pyroptosis during the acute phase of TBI. Therefore, we performed a time-effect study for damage on post-TBI sensory-motor deficits and motor dysfunctions, which has significantly higher mNSS score and minimum Rotarod test score at $24 \mathrm{~h}$. We found that acute injury significantly reduced neuron functional deficits as early as $24 \mathrm{~h}$ post-TBI. For instance, a previous study using this murine TBI model observed higher neurological deficits at $24 \mathrm{~h}$ than at $72 \mathrm{~h}$ and found that brain injury is most pronounced after $24 \mathrm{~h}$ [26]. Inflammatory correlation factors are also detected in the acute phase. A range of anti-inflammatory cytokines and pro-inflammatory cytokine secretion were all increased and continued high secretion from 24 to $48 \mathrm{~h}$, such as TGF- $\beta$, IL-10, IFN- $\gamma$, IL-1 $\beta$, and IL-18. Simultaneously, mRNA of the upstream inflammatory regulator TLR4, MyD88, and NLRP3 are also highly expressed at $24 \mathrm{~h}$ post-TBI. Apoptosis and immunostaining of the damaged cortex further validated our murine TBI model. There was significant apoptosis, expression of caspase-1, caspase-11, and GSDMD proteins around the contusion site, and blood LDH release at $24 \mathrm{~h}$ post-TBI. These data suggest that pyroptosis is likely involved in neuroinflammation with TBI. Apoptoses mediated by caspase-induced cleavage events are key features of pyroptosis [27]. Adamczak et al. verify neuronal pyroptosis 

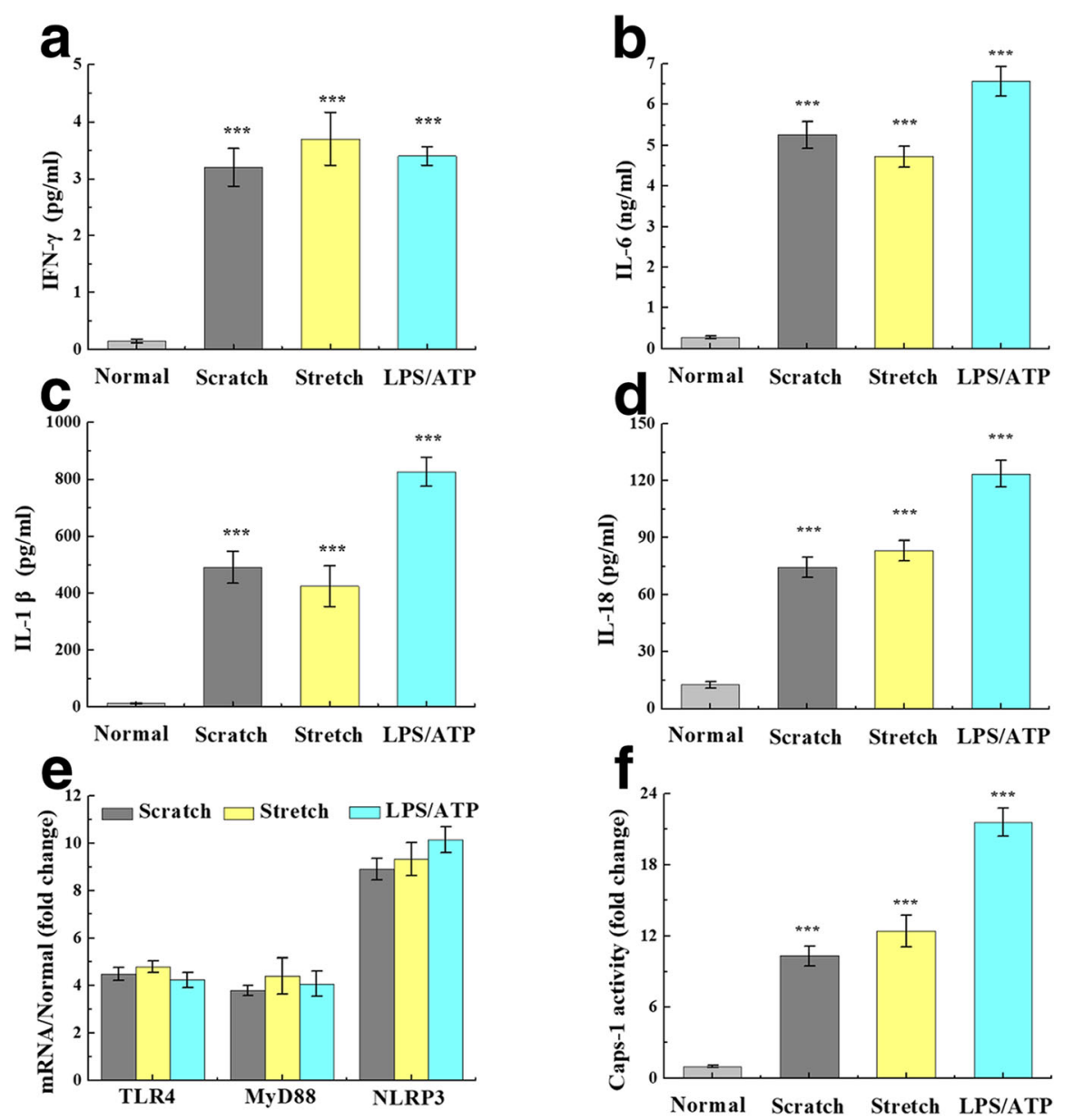

g

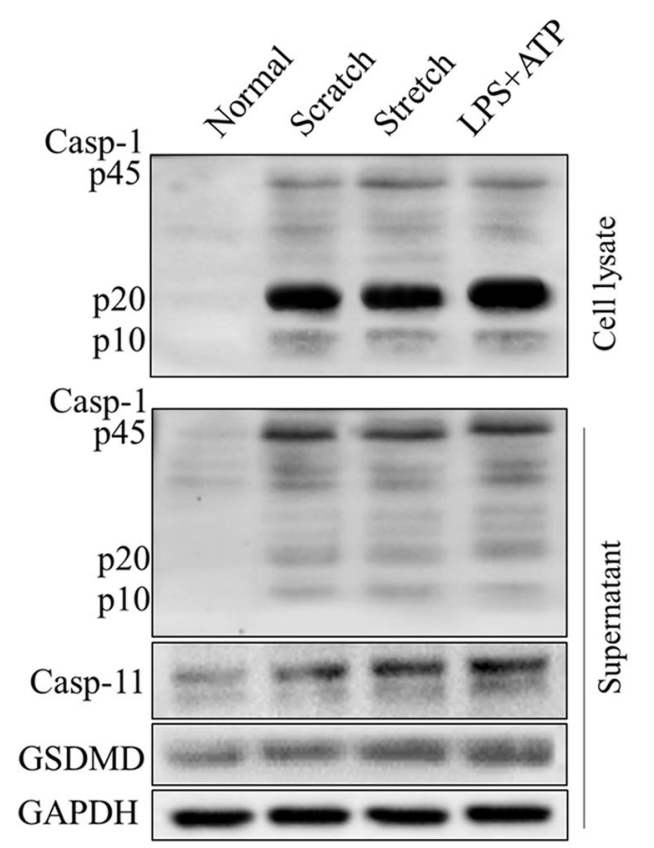

Fig. 6 (See legend on next page.) 
(See figure on previous page.)

Fig. 6 Neuron injury-induced inflammation and pyroptosis. Relative to the control group (cultured primary neuron without mechanical or chemical treatment), scratch, stretch, and LPS/ATP stimulation induced significant increases in pro-inflammatory cytokines: a IFN- $\gamma$, b IL-6, c IL-1 $\beta$, and d IL-18. e mRNA expression of TLR4, MyD88, and NLRP3 after scratch, stretch, and LPS/ATP treatments. Expression of these transcripts increased by 4.5-fold, 4.0-fold, and 9.0-fold, respectively, compared to control neurons. f Caspase-1 activity was enhanced at 10.3-fold, 12.4-fold, and 21.6-fold following scratch, stretch, and LPS/ATP stimulation, compared to the control group. $\mathbf{g}$ Cell lysate and culture supernatant were collected; pyroptosis-related protein expression was detected by WB after scratch, stretch, and LPS/ATP stimulation in cultured primary neuron. Caspase-1 and its fragments (p10 and p20) leaked to the culture supernatant. The histogram was used to analyze protein expression in the cell lysate. Data are presented as the mean \pm SEM. ${ }^{* *} p<0.001$ versus normal group; one representative experiment of five was shown

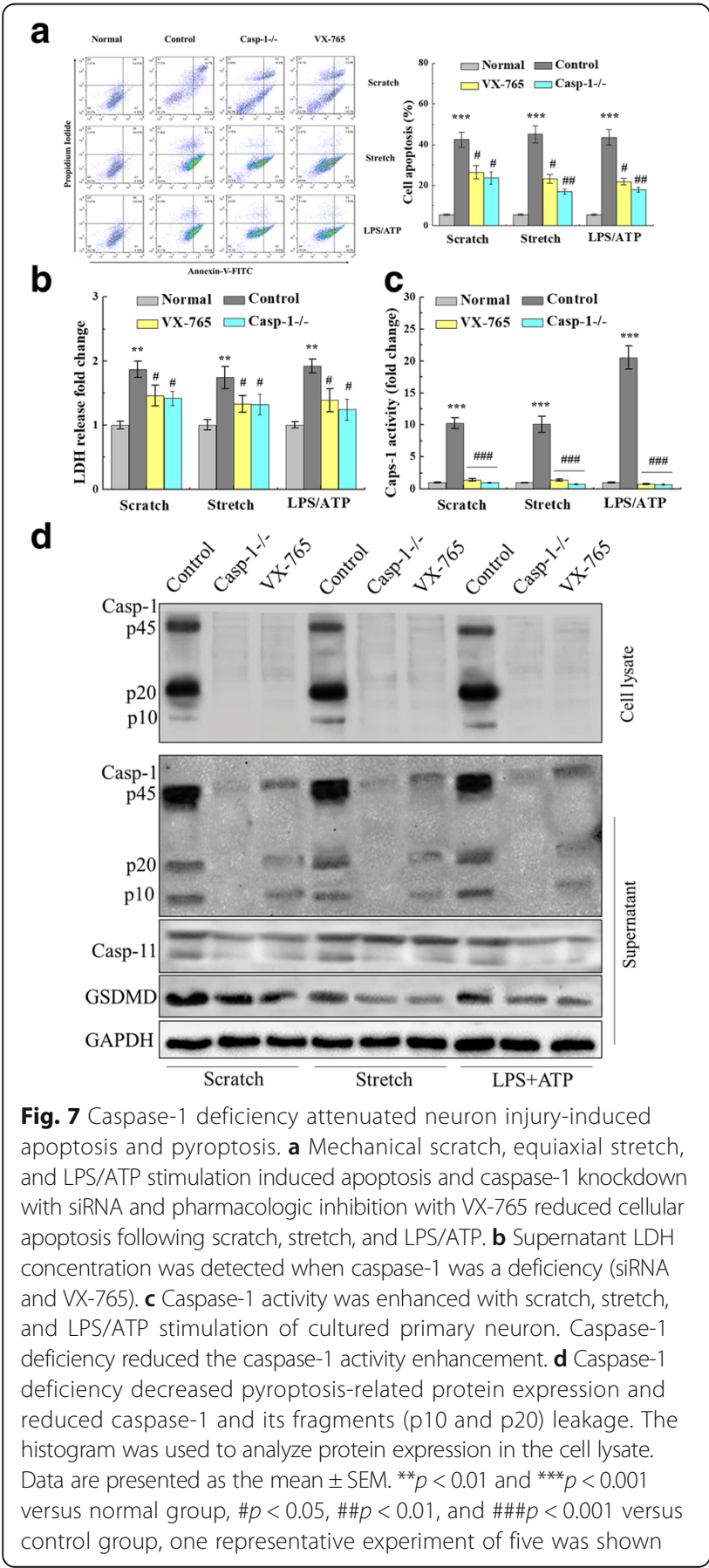

which is mediated by the AIM2 inflammasome, is an important cell death mechanism during the CNS infection and injury [28]. NLRP3 inflammasome plays a key role in the secondary phase of TBI, and lack of the NLRP3 inflammasome will improve recovery following TBI [29-31]. AIM2 and NLRP3 as sensors, they are involved in activation of caspase-1 which catalyze neuroinflammation and pyroptosis. However, we directly demonstrate whether pyroptosis involves the acute phase of TBI and whether caspase-1 deficiency will reduce neuroinflammation and neuron injury in vivo and in vitro.

Pyroptosis drives by noncanonical and canonical inflammasomes [32]. In the process of pyroptosis, Toll-like receptor (TLR)- and/or interferon (IFN)-mediated priming upregulates the expression of guanylate binding proteins critical for bacterial vacuole lysis and/or patternassociated molecular pattern (PAMP, including LPS and bacterial DNA) exposure, sensors (NLRP3, caspase-1, caspase-11), and cytokine precursor (pro-IL-1 $\beta$, pro-IL-18) $[33,34]$. The ability of inflammatory caspases to promote cell lysis involves the cleavage of GSDMD to promote the formation of membrane pores [35]. Membrane disruption leads to pyroptosis lytic cell death, releasing alarmins and pro-inflammatory cytokines [36, 37]. Noncanonical inflammasome also controls caspase- 1 activation mediated by the NLRP3 canonical inflammasome through pannexin-1 and GSDMD cleavages in a cell-intrinsic manner involving $\mathrm{K}^{+}$efflux [38-40]. Furthermore, some studies have shown that the pyroptosis is usually along with the released cytoplasmic LDH [41, 42]. All those suggest that pyroptosis involves in the acute phase of TBI, through increasing caspase-1 activity, caspase-11, GSDMD expression, and blood LDH release. While in caspase- $1^{-1-}$-TBI mice, caspase-1 deficiency impels pyroptosis decay, less LDH release, neuroinflammation, neurological deficits, and neuronal death in the acute phase.

On the cell level, we stimulated primary neuron through scratch, stretch, or LPS/ATP, to discuss the mechanism of pyroptosis on TBI. Mechanical scratch can directly damage the neurons; inflammatory cytokines in damaged neurons may release rapidly and thus affect the activity of other normal neurons. As for the mechanical stretch, it is commonly used to study the biomechanical stimulation of cardiovascular disease and bone-related 


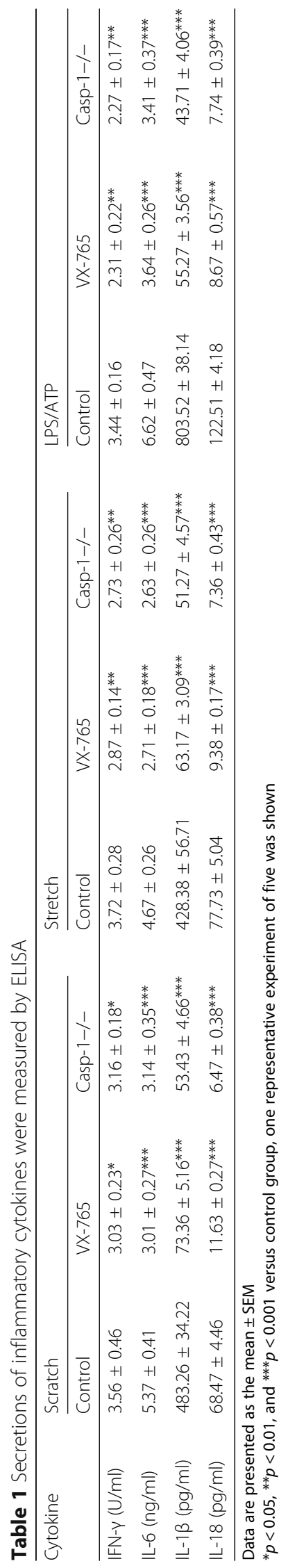


diseases [43, 44]. The neuron/axles may be damaged under equiaxial stretch (12\% strain, $1.0 \mathrm{~Hz}$ frequency) for $4 \mathrm{~h}$. In some reports, LPS usually has been used to induce inflammation and pyroptosis of macrophages [23, 36]. In this work, we tried to incur pyroptosis by LPS/ATP in the primary neuron. Scratch or stretch and LPS/ATP, all caused neuron damage, neuron apoptosis, LDH leakage, and a lot of inflammatory cytokine release, and pyroptosisrelated proteins were also triggered. Caspase is a protease involved in cell apoptosis, with more than ten subtypes. Caspase-1 regulates cell apoptosis by shearing Bcl-XL and the inflammatory response of related cytokines which are mediated by the shear of the precursors of cytokines [45]. Caspase- 1 is the unique caspase that produces active cytokines in the precursors of IL-1 $\beta$ and IL-18 [19]. Caspase-11 shears $45 \mathrm{kd}$-caspase- 1 precursor protein to produce $\mathrm{p} 10$ and p20 fragments, which form a heterogenous dimer and then form a tetrameric active enzyme [46]. In the case of caspase- 1 suppression, caspase- 1 activity was significantly inhibited, it weakens the injure and LDH leakage of neurons which induced by scratch, stretch, or LPS/ATP, and it decreased the expression or the release of inflammatory factors. Although previous studies had suggested that caspase- 1 was the main enzyme implicated in the cleavage of pro-IL-1 $\beta$ and pro-IL-18 into the biologically active cytokine, caspase- 1 and its fragments (p10 and p20) became less and mature of IL- $1 \beta$ and IL- 18 reduced when caspase- 1 was suppressed in the primary neuron. In the meantime, caspase-1 suppression aroused decrease of GSDMD, which formed large pores in the membrane that drive swelling and membrane rupture. Ultimately, caspase- 1 deficiency reduced secretion of mature IL-1 $\beta$ and IL-18, which abated neuroinflammation [47].

Our results align with what is known about the role of caspase-1 in other brain injury responses. Previous researches show that caspase- $1 \mathrm{KO}$ mice and transgenic mice expressing a dominant-negative caspase- 1 construct are partially resistant to cerebral disease and stroke. In the permanent stroke model, the proteolytic activity of caspase- 1 is increased $30 \mathrm{~min}$ after occlusion with a second wave of activation $12 \mathrm{~h}$ later [48]. The increase of caspase- 1 expression has been described in neurons and astrocytes after thromboembolic stroke and observed later in microglia (24 h post-stroke) [49]. Other studies have demonstrated the role of caspase- 1 after stroke using transgenic mice. Caspase-1 KO mice and mice expressing dominant-negative caspase- 1 exhibit a reduction of brain damage relative to wild type after experimental stroke [50]. Moreover, intracerebroventricular administration of caspase-1 inhibitors provides protective effects in experimental stroke models [51, 52]. Beneficial effects of caspase-1 inhibition are also observed in a model of oxygen and glucose deprivation in rat organotypic hippocampal slices [53]. Some studies indicate that the caspase-1
mRNA expression in the cortex was significantly increased in $\mathrm{AD}$ patients [54]. Altogether, these studies as well as our study show that caspase- 1 is a key regulator of underlying mechanisms of brain injury and disease.

Our study experimentally demonstrates that TBI induces pyroptosis, and caspase- 1 deficiency reduces neuroinflammation and neuronal damage in the acute phase. These findings may provide strategies for clinical treatment of TBI and other neuroinflammatory conditions. There is an increasing realization that neuroinflammation occurs within the whole gamut of the central nervous system pathologies [55]. Regulating inflammation to control TBI damage is possible through drug treatments [56]. NLRP3 inflammasome and interleukin-1 receptor antagonist treatments have been used to mitigate neuroinflammation after TBI [30,57]. These treatments were able to alleviate the nerve damage and promote neurological recovery after TBI. Electro-acupunctureinduced TLR4 signaling inhibition has been used to promote hippocampal neurogenesis and neurological recovery post-trauma [58].

\section{Conclusion}

Overall, our findings indicate that TBI induces pyroptosis, and caspase- 1 is a significant player in neuroinflammation. The caspase- 1 deficiency drives an anti-inflammatory response in the injured cortex, and depressed pyroptosis alleviates some of the neuroinflammation and neurological deficits involved in the acute phase of TBI. Therefore, inhibition of caspase- 1 may alleviate the severity of TBI and improve the efficacy of these other blocking agents.

\section{Additional files}

Additional file 1: Table S1. Primers of caspase-1 for RT-qPCR. (DOCX $15 \mathrm{~kb}$ ) Additional file 2: Figure S1. Caspase-1 mRNA expression level in the primary neuron. The primary neuron was infected with caspase-1 shRNA (m) lentiviral particles (Santa Cruz, sc-29922-V) according to the manufacturer's protocol (Santa Cruz Biotechnology, Inc.). At $72 \mathrm{~h}$ after infection, The RNA efficiency was determined by qRT-PCR. Caspase-1 mRNA was significantly reduced by RNAi. Data were represented as means \pm SEM, one representative experiment of four was shown. ${ }^{* * *} p<0.001$, versus control. (DOCX $24 \mathrm{~kb}$ )

Additional file 3: Figure S2. Uncropped blots for the pro-form and cleaved fragments of caspase-1 in figures. (DOCX $877 \mathrm{~kb}$ )

Additional file 4: Figure S3. VX-765 neurotoxicity detected by CKK8 assay In order to demonstrate the reduction in the pro-form of caspase-1 levels by VX-765 was not an artifact due to direct VX-765 neurotoxicity, a VX-765 alone and a vehicle control for VX-765 treated with neuron. The primary neuron was seeded in 96-well according to the primary neuronal cultures. At 10 days, cells were supplied with a fresh medium and treated with VX-765 $(0,1,5$, and $10 \mu \mathrm{M})$. After $24 \mathrm{~h}$ of cultivation respectively, then $10 \mu \mathrm{l}$ of the CCK8 reaction solution (Dojindo Laboratories) was added to each well. After $4 \mathrm{~h}$ incubation at $37^{\circ} \mathrm{C}$ in the $5 \% \mathrm{CO} 2$ incubator, the absorbance at $450 \mathrm{~nm}$ was measured by Thermo MK3 (Thermo Scientific). The primary neuron was supplied with a fresh medium and treated with VX-765 at 0, 1, 5, and $10 \mu \mathrm{M}$ (DMSO as the menstruum). After $24 \mathrm{~h}$ of cultivation respectively, neurotoxicity was detected by CCK8 assay. Data were represented as means $\pm \mathrm{SEM}$, one representative experiment of five was shown. (DOCX $43 \mathrm{~kb}$ ) 


\section{Abbreviations}

AD: Alzheimer disease; ANOVA: Analysis of variance; Aß: Amyloid-beta peptide; Bach: BTB and CNC homology; BCA: Bicinchoninic acid; DAB: 3,3'-Diaminobenzidine solution; DAPI: 4',6-Diamidino-2-phenylindole DNA: Deoxyribonucleic acid; ELISA: Enzyme-linked immunosorbent assay; Erk: Extracellular regulated protein kinases; FITC: Fluorescein isothiocyanate; GAPDH: Glyceraldehyde-3-phosphate dehydrogenase; GSDMD: Gasdermin D; HRP: Avidin-biotin horseradish peroxidase; IFN: Interferon; IL: Interleukin; KO: knockout; LPS: Lipopolysaccharide; mNSS: Modified neurological severity score; MyD: Myeloid differentiation primary-response gene; NLRP: Nucleotide-binding oligomerization domain, leucine rich repeat, and pyrin domain containing; Nrf: NF-E2-related factor; PBS: Phosphate-buffered saline; PCR: Polymerase chain reaction; PI: Propidium iodide; Rick: Receptor-interacting serine-threonine kinase; Rip: Receptor-interacting protein; RNA: Ribonucleic acid; SAH: Stable single a-helices; SD: Standard deviation; siRNA: Small interfering RNA; TBI: Traumatic brain injury; TGF: Transforming growth factor; TLR: Toll-like receptors; TNF: Tumor necrosis factor; TUNEL: Terminal deoxynucleotidyl transferase-dUTP nick end labeling; WB: Western blotting; WT: Wide-type

\section{Acknowledgements}

The authors thank the professional biomedical editor Dr. Marie Barabas (PhD) for the help with language revising.

\section{Funding}

This work was supported by the Development Funding of Shaanxi Fourth People Hospital (2017SY-006).

\section{Availability of data and materials}

Data supporting the conclusions of this article are presented in the manuscript.

\section{Authors' contributions}

WL and YHC conceived and designed the study. JM and FFB developed the methodology. CCC, HL, and LJZ carried out the experiments. YHC and FFB interpreted the results. MFW performed the data analysis and prepared the figures. YHC wrote the manuscript. $W L$ and $Y H C$ reviewed and revised the manuscript and supervised the study. All authors read and approved the manuscript.

\section{Ethics approval}

All experimental procedures used in this study were approved by the Animal Ethics Committee of the Shaanxi Forth People Hospital (Shaanxi, China).

\section{Consent for publication}

Not applicable.

\section{Competing interests}

The authors declare that they have no competing interests.

\section{Publisher's Note}

Springer Nature remains neutral with regard to jurisdictional claims in published maps and institutional affiliations.

\section{Author details}

${ }^{1}$ Department of Medical Science Research Center, Peihua University, Xi'an 710125, People's Republic of China. ${ }^{2}$ Department of Medical Science Research Center, Shaanxi Fourth People Hospital, Xi'an 710043, People's Republic of China. ${ }^{3}$ Department of Orthopedics, Jilin University Second Hospital, Changchun 8974617, People's Republic of China.

Received: 7 November 2017 Accepted: 31 January 2018

Published online: 19 February 2018

\section{References}

1. Van Horn JD, Bhattrai A, Irimia A. Multimodal imaging of neurometabolic pathology due to traumatic brain injury. Trends Neurosci. 2017:40:39-59.

2. Dang B, Chen W, He W, Chen G. Rehabilitation treatment and progress of traumatic brain injury dysfunction. Neural Plast. 2017;2017:1582182.

3. Runyan DK. The challenges of assessing the incidence of inflicted traumatic brain injury: a world perspective. Am J Prev Med. 2008;34(4 Suppl):S112-5.
4. Hemphill MA, Dauth S, Yu CJ, Dabiri BE, Parker KK. Traumatic brain injury and the neuronal microenvironment: a potential role for neuropathological mechanotransduction. Neuron. 2015;85:1177-92.

5. Roozenbeek B, Maas Al, Menon DK. Changing patterns in the epidemiology of traumatic brain injury. Nat Rev Neurol. 2013;9:231-6.

6. Julien J, Joubert S, Ferland MC, Frenette LC, Boudreau-Duhaime MM, MaloVéronneau $L$, et al. Association of traumatic brain injury and Alzheimer disease onset: a systematic review. Ann Phys Rehabil Med. 2017;60(5):34756.

7. Gardner AJ, Shih SL, Adamov EV, Zafonte RD. Research frontiers in traumatic brain injury: defining the injury. Phys Med Rehabil Clin N Am. 2017;28:413-31.

8. Esterov D, Greenwald BD. Autonomic dysfunction after mild traumatic brain injury. Brain Sci. 2017;7(8). https://doi.org/10.3390/brainsci7080100

9. Russo MV, McGavern DB. Inflammatory neuroprotection following traumatic brain injury. Science. 2016;353:783-5.

10. Zetterberg $\mathrm{H}$, Smith DH, Blennow K. Biomarkers of mild traumatic brain injury in cerebrospinal fluid and blood. Nat Rev Neurol. 2013;9:201-10.

11. Corrigan F, Mander KA, Leonard AV, Vink R. Neurogenic inflammation after traumatic brain injury and its potentiation of classical inflammation. J Neuroinflammation. 2016:13:264.

12. Wang $Y$, Fan $X$, Tang $T$, Fan R, Zhang C, Huang Z, et al. Rhein and rhubarb similarly protect the blood-brain barrier after experimental traumatic brain injury via gp91<sup >phox</sup> subunit of NADPH oxidase/ROS/ERK MMP-9 signaling pathway. Sci Rep. 2016;6:37098.

13. Angeloni C, Prata C, Dalla Sega FV, Piperno R, Hrelia S. Traumatic brain injury and NADPH oxidase: a deep relationship. Oxidative Med Cell Longev. 2015:2015:370312

14. Rao W, Zhang L, Peng C, Hui H, Wang K, Su N, et al. Downregulation of STIM2 improves neuronal survival after traumatic brain injury by alleviating calcium overload and mitochondrial dysfunction. Biochim Biophys Acta. 2015:1852:2402-13.

15. Dobrachinski F, da Rosa GR, Sartori G, Ferreira Marques N, Zemolin AP, Almeida Silva LF, et al. Regulation of mitochondrial function and glutamatergic system are the target of guanosine effect in traumatic brain injury. J Neurotrauma. 2017:34:1318-28.

16. Hiebert JB, Shen $Q$, Thimmesch AR, Pierce JD. Traumatic brain injury and mitochondrial dysfunction. Am J Med Sci. 2015;350:132-8.

17. Janowitz T, Menon DK. Exploring new routes for neuroprotective drug development in traumatic brain injury. Sci Transl Med. 2010;2:27rv1.

18. Fink SL, Cookson BT. Caspase-1-dependent pore formation during pyroptosis leads to osmotic lysis of infected host macrophages. Cell Microbiol. 2006:8:1812-25.

19. Yuan J, Najafov A, Py BF. Roles of caspases in necrotic cell death. Cell. 2016:167:1693-04.

20. Barrington J, Lemarchand E, Allan SM. A brain in flame; do inflammasomes and pyroptosis influence stroke pathology? Brain Pathol. 2017;27:205-12

21. Mezzaroma E, Toldo S, Farkas D, Seropian IM, Van Tassell BW, Salloum FN, et al. The inflammasome promotes adverse cardiac remodeling following acute myocardial infarction in the mouse. Proc Natl Acad Sci U S A. 2011;108:19725-30.

22. Yang D, He Y, Muñoz-Planillo R, Liu Q, Núñez G. Caspase-11 requires the pannexin-1 channel and the purinergic P2X7 pore to mediate pyroptosis and endotoxic shock. Immunity. 2015;43:923-32.

23. Wu D, Pan $P$, Su X, Zhang $L$, Qin $Q$, Tan $H$, et al. Interferon regulatory factor- 1 mediates alveolar macrophage pyroptosis during LPS-induced acute lung injury in mice. Shock. 2016:46:329-38.

24. Wu H, Huang T, Ying L, Han C, Li D, Xu Y, et al. MiR-155 is involved in renal ischemia-reperfusion injury via direct targeting of FoxO3a and regulating renal tubular cell pyroptosis. Cell Physiol Biochem. 2016;40:1692-05.

25. Li R, Zhang LM, Sun WB. Erythropoietin rescues primary rat cortical neurons from pyroptosis and apoptosis via Erk1/2-Nrf2/Bach1 signal pathway. Brain Res Bull. 2017;130:236-44

26. Xu X, Gao W, Cheng S, Yin D, Li F, Wu Y, et al. Anti-inflammatory and immunomodulatory mechanisms of atorvastatin in a murine model of traumatic brain injury. J Neuroinflammation. 2017;14:167.

27. Sharma D, Kanneganti TD. The cell biology of inflammasomes: mechanisms of inflammasome activation and regulation. J Cell Biol. 2016:213:617-29.

28. Adamczak SE, de Rivero Vaccari JP, Dale G, Brand FJ, Nonner D, Bullock MR, et al. Pyroptotic neuronal cell death mediated by the AIM2 inflammasome. J Cereb Blood Flow Metab. 2014;34(4):621-9. 
29. Liu HD, Li W, Chen ZR, Hu YC, Zhang DD, Shen W, et al. Expression of the NLRP3 inflammasome in cerebral cortex after traumatic brain injury in a rat model. Neurochem Res. 2013;38(10):2072-83.

30. Irrera N, Pizzino G, Calò M, Pallio G, Mannino F, Famà F, et al. Lack of the Nlrp3 inflammasome improves mice recovery following traumatic brain injury. Front Pharmacol. 2017:8:459.

31. Mortezaee K, Khanlarkhani N, Beyer C, Zendedel A. Inflammasome: its role in traumatic brain and spinal cord injury. J Cell Physiol. 2017; https://doi.org/10.1002/jcp.26287.

32. de Zoete MR, Palm NW, Zhu S, Flavell RA. Inflammasomes. Cold Spring Harb Perspect Biol. 2014;6:a016287.

33. Wellington M, Koselny K, Sutterwala FS, Krysan DJ. Candida albicans triggers NLRP3-mediated pyroptosis in macrophages. Eukaryot Cell. 2014;13:329-40.

34. Liu Z, Gan L, Xu Y, Luo D, Ren Q, Wu S, et al. Melatonin alleviates inflammasome-induced pyroptosis through inhibiting NF-KB/GSDMD signal in mice adipose tissue. J Pineal Res. 2017;63 https://doi.org/10.1111/jpi.12414.

35. Liu X, Zhang Z, Ruan J, Pan Y, Magupalli VG, Wu H, et al. Inflammasome-activated gasdermin D causes pyroptosis by forming membrane pores. Nature. 2016;535:153-8.

36. Chen $\mathrm{Q}$, Jin $Y$, Zhang $\mathrm{K}$, Li H, Chen W, Meng G, et al. Alarmin HNP-1 promotes pyroptosis and IL-1 $\beta$ release through different roles of NLRP3 inflammasome via P2X7 in LPS-primed macrophages. Innate Immun. 2014;20:290-00.

37. Ding J, Wang K, Liu W, She Y, Sun Q, Shi J, et al. Pore-forming activity and structural autoinhibition of the gasdermin family. Nature. 2016:535:111-6.

38. Kayagaki N, Warming S, Lamkanfi M, Vande Walle L, Louie S, Dong J, et al. Non-canonical inflammasome activation targets caspase-11. Nature. 2011;479:117-21

39. Lin QR, Li CG, Zha QB, Xu LH, Pan H, Zhao GX, et al. Gossypol induces pyroptosis in mouse macrophages via a non-canonical inflammasome pathway. Toxicol Appl Pharmacol. 2016:292:56-64.

40. Albalawi F, Lu W, Beckel JM, Lim JC, McCaughey SA, Mitchell CH. The P2X7 receptor primes IL-1 $\beta$ and the NLRP3 inflammasome in astrocytes exposed to mechanical strain. Front Cell Neurosci. 2017;11:227.

41. Okondo MC, Johnson DC, Sridharan R, Go EB, Chui AJ, Wang MS, et al. DPP8/9 inhibition induces pro-caspase-1-dependent monocyte and macrophage pyroptosis. Nat Chem Biol. 2017;13(1):46-53.

42. DiPeso L, Ji DX, Vance RE, Price JV. Cell death and cell lysis are separable events during pyroptosis. Cell Death Discov. 2017:3:17070.

43. Shradhanjali A, Riehl BD, Lee JS, Ha L, Lim JY. Enhanced cardiomyogenic induction of mouse pluripotent cells by cyclic mechanical stretch. Biochem Biophys Res Commun. 2017:488:590-5.

44. Yu HS, Kim JJ, Kim HW, Lewis MP, Wall I. Impact of mechanical stretch on the cell behaviors of bone and surrounding tissues. J Tissue Eng. 2016;7: 2041731415618342.

45. Sun Q, Scott MJ. Caspase-1 as a multifunctional inflammatory mediator: noncytokine maturation roles. J Leukoc Biol. 2016;100:961-7.

46. Wang $\mathrm{S}$, Miura M, Jung YK, Zhu H, Li E, Yuan J. Murine caspase-11, an ICE-interacting protease, is essential for the activation of ICE. Cell. 1998:92:501-9.

47. Kovacs SB, Miao EA. Gasdermins: effectors of pyroptosis. Trends Cell Biol. 2017:27:673-84.

48. Benchoua A, Guégan C, Couriaud C, Hosseini H, Sampaïo N, Morin D, et al. Specific caspase pathways are activated in the two stages of cerebral infarction. J Neurosci. 2001;21:7127-34.

49. Abulafia DP, de Rivero Vaccari JP, Lozano JD, Lotocki G, Keane RW, Dietrich WD. Inhibition of the inflammasome complex reduces the inflammatory response after thromboembolic stroke in mice. J Cereb Blood Flow Metab. 2009;29:534-44.

50. Schielke GP, Yang GY, Shivers BD, Betz AL. Reduced ischemic brain injury in interleukin-1 beta converting enzyme-deficient mice. J Cereb Blood Flow Metab. 1998;18:180-5.

51. Ross J, Brough D, Gibson RM, Loddick SA, Rothwell NJ. A selective, non-peptide caspase-1 inhibitor, VRT-018858, markedly reduces brain damage induced by transient ischemia in the rat. Neuropharmacology. 2007:53:638-42.

52. Rabuffetti M, Sciorati C, Tarozzo G, Clementi E, Manfredi AA, Beltramo M. Inhibition of caspase-1-like activity by Ac-Tyr-Val-Ala-Asp-chloromethyl ketone induces long-lasting neuroprotection in cerebral ischemia through apoptosis reduction and decrease of proinflammatory cytokines. J Neurosci. 2000;20:4398-04
53. Ray AM, Owen DE, Evans ML, Davis JB, Benham CD. Caspase inhibitors are functionally neuroprotective against oxygen glucose deprivation induced CA1 death in rat organotypic hippocampal slices. Brain Res. 2000;867:62-9.

54. Kaushal V, Dye R, Pakavathkumar P, Foveau B, Flores J, Hyman B, Ghetti B, Koller BH, LeBlanc AC. Neuronal NLRP1 inflammasome activation of caspase-1 coordinately regulates inflammatory interleukin-1-beta production and axonal degeneration-associated caspase- 6 activation. Cell Death Differ. 2015;22:1676-86.

55. Helmy A, Carpenter KL, Menon DK, Pickard JD, Hutchinson PJ. The cytokine response to human traumatic brain injury: temporal profiles and evidence for cerebral parenchymal production. J Cereb Blood Flow Metab. 2011;31:658-70.

56. Thelin EP, Tajsic T, Zeiler FA, Menon DK, Hutchinson PJA, Carpenter KLH, et al. Monitoring the neuroinflammatory response following acute brain injury. Front Neurol. 2017;8:351.

57. Sun M, Brady RD, Wright DK, Kim HA, Zhang SR, Sobey CG, et al. Treatment with an interleukin-1 receptor antagonist mitigates neuroinflammation and brain damage after polytrauma. Brain Behav Immun. 2017; [Epub ahead of print].

58. Ye Y, Yang Y, Chen C, Li Z, Jia Y, Su X, et al. Electroacupuncture improved hippocampal neurogenesis following traumatic brain injury in mice through inhibition of TLR4 signaling pathway. Stem Cells Int. 2017;2017:5841814.

\section{Submit your next manuscript to BioMed Central and we will help you at every step:}

- We accept pre-submission inquiries

- Our selector tool helps you to find the most relevant journal

- We provide round the clock customer support

- Convenient online submission

- Thorough peer review

- Inclusion in PubMed and all major indexing services

- Maximum visibility for your research

Submit your manuscript at www.biomedcentral.com/submit 